\title{
EMOCIONES DE LOS ADOLESCENTES INTERNADOS EN UN CENTRO DE ORIENTACIÓN JUVENIL. CÓRDOBA - COLOMBIA
}

\author{
EMOTIONS OF ADOLESCENTS INSIDE IN A YOUTH GUIDE CENTER. \\ CORDOBA - COLOMBIA
}

\author{
Concepción E Amador $\mathrm{A}^{1}$. Angélica Avendaño $\mathrm{V}^{2}$. Karina Caldera $\mathrm{O}^{3}$ \\ Recibido para publicación: Febrero 16 de 2017 - Aceptado para publicación: Mayo 10 de 2017
}

\begin{abstract}
RESUMEN
Objetivo: Identificar las emociones que tienen los adolescentes acerca de su internación en el Centro de Orientación Juvenil. Materiales y métodos. Utilizando metodologíascualitativas, seabordóprevioconsentimiento informado de la institución, de los cuidadores profesionales y de los 16 adolescentes internados se aplicó mediante entrevista directa un instrumento semiestructurado validado por la experiencia y un diario de campo para consignar las relaciones de las respuestas verbales con las no verbales. Mediante análisis documentado las 34 expresiones semánticas se agruparon por semejanza de contenidos hasta obtener las 4 categorías de interés para el estudio. Resultados. Los adolescentes tienen emociones positivas con respecto a su internación, consideran que ésta le ofrece seguridad personal, confianza y satisfacción de sus necesidades básicas cotidianas. En la categoría de relaciones interpersonales con los pares propia de la esfera personal-social del adolescente se identificó la presencia de emociones negativas o displacenteras entre quienes tenían historia personal de carencias afectivas; por el contrario entre quienes tenían antecedentes afectivos previos manifestaron mejores relaciones con los pares a quienes llegan a considerar como parte de sus familias. Conclusiones. La internación de adolescentes puede alterar la dimensión emocional en el campo personalsocial de quienes no han tenido vínculos afectivos previos. Recomendaciones. Retomar los criterios para sustraer de los hogares sustitutos a los adolescentes que tienen comportamientos dentro de lo esperado para la edad. Involucrar la dimensión personal-social dentro de los logros de desarrollo previos a la emancipación.
\end{abstract}

Palabras clave: Adolescencia, Internación, Emoción.

\begin{abstract}
Objective: To identify the emotions that adolescents have about your stay in the Youth Guidance Center. Materials and methods. Using qualitative methods, consent was addressed was informed of the institution, professional caregivers and los16 hospitalized adolescents was applied by direct interview a semistructured instrument validated by experience and a field diary to record the relations of verbal responses with no verbal. Documented analyses using the 34 semantic expressions were grouped by similarity of content until 4 categories of interest to the study. Results. Teenagers have positive emotions regarding your stay; they consider this offer personal security, confidence and satisfaction of their daily basic needs. In the category of interpersonal relationships with peers own personal-social sphere adolescent the presence of negative or unpleasurable emotions among those with personal history of emotional deprivation identified; on the contrary among those with affective previous history they showed better relationships with peers who come to consider as part of their families. Conclusions. The internment of adolescents apparently alters the emotional dimension in the social field staff who have not had previous emotional ties. Recommendations. Retaking the criteria for subtracting from foster care to adolescents with behavior within expected for age. Involve personalsocial dimension in development achievements before emancipation.
\end{abstract}

Key words: Adolescents, Internment, Emotion.

\footnotetext{
${ }^{1}$ Enfermera, Esp. Y Msc, en Enfermería, Profesor Titular, Departamento de Enfermería, Facultad Ciencias de la Salud, Universidad de Córdoba, Montería, Colombia. concepcionamador@correo.unicordoba.edu.co

${ }^{2}$ Enfermera, Facultad Ciencias de la Salud, Universidad de Córdoba, Montería, Colombia.

${ }^{3}$ Enfermera, Facultad Ciencias de la Salud, Universidad de Córdoba, Montería, Colombia.
} 


\section{INTRODUCCIÓN}

La adolescencia ha sido definida y considerada como "una etapa de cambios que influyen de manera determinante en la personalidad del individuo en su edad adulta y oscila entre los 10 y 20 años". Durante este ciclo pueden presentarse transformaciones a nivel biopsicosocial, que también pueden conllevar a las crisis normativas y/o no normativas las cuales están determinadas por la búsqueda de independencia, propia en esta etapa (1).

Esta etapa siempre representa una crisis, tanto biológica como psicológica. Este período se caracteriza por un aumento de las emociones, cuyas manifestaciones varían en cada individuo que se encuentre en esta etapa. Una emoción es un estado afectivo que experimenta el ser humano, una reacción subjetiva al ambiente que viene acompañada de cambios orgánicos (fisiológicos y endocrinos) de origen innato, los cuales pueden ser influidos por la experiencia como son las tendencias de respuesta adaptativas, la experiencia subjetiva, el procesamiento de la información y similares que surgen ante la evaluación de algún acontecimiento (2).

El estudio de la clasificación de las emociones las organiza como negativas entre ellas el miedo, ira, asco, tristeza, y demás; por su parte la alegría, orgullo, satisfacción, esperanza, fluidez, elevación, entre otros son clasificadas como emociones positivas (3).

Las emociones positivas o placenteras se pueden asociar a tendencias cognitivas de pensamientos pro activos en una serie de respuestas claras y específicas, que pueden ser moduladas a voluntad del individuo; en tanto que las emociones negativas no se asocian con pensamientos positivos, creativos o de avances; pero emociones negativas o displacenteras no siguen una secuencia cognitiva por tanto no aportan a funciones intrínsecas del desarrollo de la dimensión personal social de la salud (3).

El ingreso de un individuo a una institución o establecimiento con diferente finalidad, es definido como internación; un niño o adolescente ingresa a centros de orientación juvenil o de protección especial, si se encuentra en una situación irregular (4); en Colombia estas condiciones están descritas en el Código del Menor. Asímismo, las medidas que se tomaran según el caso.

La institucionalización de Niños, Niñas y Adolescentes (NNA) en condición de vulneración de sus derechos fundamentales se convierte en una solución parcial del Estado para cumplir los mandatos constitucionales de primacía de los derechos de los NNA contenidos en la Constitución Política Nacional de la República de Colombia compromiso que se da sobre todo en un régimen modulado de control social y alimenticio; sin embargo la misma internación que salvaguarda los derechos fundamentales de los NNA puede ir generando con el transcurrir del tiempo otro tipo de falencias de utilidad para el desarrollo social y humano como la interacción social, debido a la privación de modelos y de figuras materna o paterna que otorguen amor y seguridad.

Si el conjunto de estas dimensiones emocionales, de importancia para el desarrollo social no se encuentran adecuadamente desarrolladas en el adolescente, pueden conducir a que éste en la adultez tenga dificultades para expresar sus sentimientos y pensamientos, y le impiden desarrollar esquemas de acción con responsabilidad y de compromiso $(3,5)$; por lo cual se realizó el estudio con el fin de identificar las emociones que tienen los jóvenes que están en situación de internación institucional para protección o restitución de sus derechos. 


\section{MATERIALES Y MÉTODOS}

Con el objetivo de identificar las respuestas emocionales de los adolescentes ante su internación en un Centro de Orientación Juvenil, se realizó un estudio de tipo cualitativo, en una población constituida por adolescentes internados en un Centro de Orientación Juvenil localizado en la Costa Caribe de Colombia (SA) que sirvió de escenario al estudio.

La muestra estuvo formada por 16 adolescentes de uno u otro sexo, con edades en el rango de 13 a 19 años; la unidad de análisis fue la respuesta emocional del adolescente a la internación. Se utilizó muestreo no probabilístico por conveniencia. Para poder participar en la investigación, el (la) adolescente debía cumplir los criterios que se enuncian: edad entre 13 y 19 años, internados al momento del estudio en el Centro de Orientación Juvenil, periodo mínimo un mes de internación.

La información se recopiló a través de entrevista semiestructurada creada y diligenciada por el equipo investigador; la cual fue grabada en audio. Al instrumento se le realizó previa prueba piloto y validación por el personal del Centro de Orientación Juvenil.

La entrevista estuvo formada por 15 preguntas elaboradas con la finalidad que el adolescente manifestara sus emociones acerca de diferentes situaciones que experimentan debido a su internación (ambiente interno y externo); cinco de las quince preguntas fueron abiertas (preguntas 1, 2, 4, 5, 13), las respuestas fueron categorizadas según grados de semejanza de los contenidos semánticos; de los 34 contenidos semánticos obtenidos, luego de su agrupación por semejanzas se identificaron 4 categorías de interés para el tema estudiado.

Asimismo, mediante un diario de campo se tomó en consideración las respuestas no verbales o corporales de cada participante ante las preguntas.
Durante todo el proceso investigativo, se respetaron y aplicaron los parámetros contenidos en la Resolución 8430 de 1993 del Ministerio de Salud, relacionadas con las Normas científicas, técnicas y administrativas para la investigación con seres humanos. Debido a que las preguntas tenían la posibilidad de movilizar emociones negativas, se clasificó con "riesgo ético mínimo". El control del riesgo se hizo con apoyo psicoafectivo por profesional, consejería y educación con el apoyo de los profesionales respectivos del centro; solo dos de los participantes requirieron este tipo de control.

Además, se cumplió con el respeto y aplicación de los principios de la ética de la investigación contenidos en las Declaraciones de Helsinki I y II en particular la confidencialidad, beneficencia, participación voluntaria y el consentimiento informado el cual se obtuvo directamente de los participantes previa explicación de los objetivos y alcances de su participación. En salvaguarda de la confidencialidad los nombres de los participantes fueron remplazados en todo el proceso por colores.

\section{RESULTADOS}

Emociones Placenteras y Displacenteras: las emociones son consideradas como algo personal e intimo a lo que muy pocas personas tienen acceso (3), al respecto, de lo antes dicho, se identificó entre los adolescentes internados para protección de sus derechos fundamentales, la presencia de ciertas expresiones que muestran su sentir emocional con respecto a una variedad de situaciones, así:

\section{- ¿Considera justificable su internación?}

"No, porque mi problema no tiene importancia" (blanco)

"Digamos que si" -expresión gestual de indiferencia- (azul) 
- ¿Qué crees que estarías haciendo fuera del centro de orientación?

"No estaría vivo" (gris)

"Yo no estaría ni para echar el cuento" (fucsia)

"Estaría en mi casa con mi mamá" (violeta)

"Estuviera con mi familia sustituta" (azul)

Ante estas expresiones semánticas de sus emociones en la internación, puede observarse el predominio de respuestas que marcan posiciones de indiferencia con respecto hacia sí mismos, hacia su ser, expresiones que indican que vivencias pasadas les generaron temor sobre su propia vida y supervivencia como los casos que fueron identificados como "gris" y "fucsia".

Estas expresiones así mismo son simbólicas de la emoción positiva o placentera que les da la percepción de seguridad y confianza durante su estancia en el Centro de Orientación Juvenil; los adolescentes entrevistados reconocen en el Centro de Orientación a un ente que suple sus necesidades básicas del cual reciben apoyo, así como los aportes de los miembros del equipo interdisciplinario que allí labora.

Las emociones pueden presentarse de manera externa mediante reacciones positivas 0 negativas, relacionadas con ciertas situaciones. Sin embargo, las respuestas emotivas autóctonas o intrapersonales de los adolescentes en condición de internación fueron clasificadas como displacenteras porque hacen parte de un conjunto de respuestas compatibles con trastornos afectivos como la depresión.

Tales emociones displacenteras fueron el consenso entre los adolescentes internados incluso en aquellas que en apariencia podían ser calificadas como positivas como la expresada por "azul" y "violeta"; las cuales fueron incorporadas en este conjunto debido a las claras inconsistencias entre el lenguaje gestual y el verbal; en estos casos se dio prioridad a la interpretación del lenguaje corporal y entonación vocal y no solo a la expresión verbal, es decir que en ambos casos se identificó indiferencia.

También en estas mismas expresiones que muestran la indiferencia emocional del adolescente en las expresiones de "Azul" y "Blanco" en las que el sentido la expresión semántica de la idea se acompaña de muestras corporales y de entonación compatibles con la indiferencia afectiva. (5)

Se analizó de forma cualitativa el anhelo contenido en las expresiones semánticas, según las cuales los individuos identificados como "Violeta" y "Azul" desean estar al interior de un vínculo familiar propio o sustituto que los provea del afecto (4). Estas expresiones proyectan las falencias de la pertenencia a grupos filiales a los cuales ha pertenecido en el transcurrir de su historia de vida.

En este mismo sentido, sería interesante discriminar o clarificar las razones por las cuales el sistema de protección del estado considera que es emocionalmente sano y pertinente que los adolescentes en protección sean internados en este tipo de instituciones; pues con este acto se les despoja del derecho a pertenecer $y$ desarrollarse en un ambiente familiar con todas las probabilidades de adaptación social y cultural que tiene esta inserción familiar.

Pues si bien, por la naturaleza de los actos de sus padres no pueden estar en su hogar, en su familia nuclear, bien podrían pertenecer a otro grupo familiar por afinidad; también se pudo identificar que en varios casos los adolescentes fueron sustraídos de familias sustitutas e internados en Centro de Orientación por asuntos o comportamientos propios de la adolescencia, es decir debido a fallas conductuales $(6,7)$.

Esta abrupta separación de una segunda familia genera profundos duelos por las repetidas pérdidas (5), las cuales afectan de manera negativa no solo las emociones del 
adolescente, sino también su comportamiento y autopercepción al alterar su inteligencia emocional afectando la salud y estabilidad mental $(4,7,8)$.

Es prudente retomar en este aspecto del análisis que en los escenarios académicos y sociales se considera que "el principal factor de protección es el familiar", debido a que la familia, el grupo con sus funciones y roles realiza de manera continua el "refuerzo de la enseñanza de las habilidades propias de la inteligencia emocional" $(6,9)$.

Relaciones con los pares. Se ha observado y sustentado que la interacción social y la pertenencia a un grupo son definitivas en el desarrollo normal de un adolescente (10), aunque el desarrollo de la convivencia de diversas personalidades pueda generar, percepciones de rechazo, duda, angustia o tristeza (11). Teniendo en cuenta lo anterior, el acto de internación en un espacio que no es propio, no es de nadie trae como consecuencia problemas de interacción con sus pares, los cuales se expresan de manera explícita e impiden que algunos de ellos se integren como familia así:

\section{- ¿Cómo es tu relación con los demás} adolescentes internados?

"Algunas compañeras me tienen rabia, a mi no me importa lo que piensen de mí, no me hablo como con tres" (Fucsia)

"Buena, a veces peleamos pero después estamos bien" (Lila)

"Buena, a algunos no les gusta mi forma de ser, pero soy yo, así soy yo" (Azul)

"Excelente, son mis amigas" (Beige)

"No tengo amigos sino compañeros. Si, si puedo contar con ellos." (Gris)

- ¿Crees que tus compañeras hacen parte de tu familia?

"Si las considero me aconsejan y no nos llevamos mal" (Beige)
"No ellas no son mi familia" (Violeta)
"Si, son como mis hermanas" (Rosado)

Es posible evidenciar el predominio del efecto de las carencias afectivas expresadas como indiferencia; hecho identificable entre quienes tienen historial de negligencia y abandono; este grupo en sus expresiones denotan anticipo de rechazo, reserva, cautela y miedo como puede evidenciarse en las expresiones de "Azul", "Gris", "Violeta", y "Fucsia", sus respuestas contienen un temor básico al miedo a ser excluido e ignorado; los contenidos de las declaraciones de estos adolescentes muestran sentimientos de rechazo, tristeza e indiferencia, que impide que desde la internación se produzcan los vínculos filiales que se esperaría de la convivencia permanente $(5,10,11)$.

Desde la salud mental y social es importante destacar, que el adolescente en internación debería aprender a manejar sus relaciones sociales de manera constructiva en la interacción continua y permanente con sus pares; sin embargo tal logro no parece estarse cumpliendo por las fragilidades de la inteligencia emocional inducida por la persistencia de emociones displacenteras ya analizadas; los adolescentes en internación "aceptan" los rechazos, las discusiones y las lejanías sin que se afecte la convivencia, porque no les importa nada es por lo anterior que se puede apreciar el aislamiento voluntario e incapacidad para intentar resolver las diferencias de carácter; entonces sus emociones de indiferencia le impiden lograr los mecanismos de ajuste a la diferencia y a la variabilidad de las personalidades presentes en todos los ámbitos sociales $(4,5,7,9)$.

En tanto que, entre los adolescentes que algún período de sus vidas, recibieron expresiones afectivas se identificaron sentimientos $y$ emociones positivas, placenteras como alegría y satisfacción, es así como las participantes "Lila", "Rosado" y "Beige" quienes manifiestan excelente interacción con sus pares, aunque 
tengan dificultades los adolescentes internados con una gama emocional placentera siempre tratan de resolverlos, colaborando para mantener un ambiente adecuado y de apoyo mutuo; los vínculos filiales que se forman en estos casos permite que los miembros del grupo sean considerarlos como parte de su familia; contribuyendo así al desarrollo más cercano a lo normal en la etapa del ciclo vital individual en que se encuentran $(3,5,10,12)$.

Podría entonces apreciarse, que las experiencias afectivas previas que se comparten en la interacción mediante relaciones emotivas y afectivas con los pares en la internación ejercen una definitiva influencia positiva sobre la gama de emociones y sobre la percepción que el adolescente internado tiene de sí mismo y de sus relaciones como persona en grupo y en sociedad (12).

\section{DISCUSIÓN}

En los ámbitos de las ciencias relacionadas con la vida emocional y la salud mental se reconoce que las emociones que se expresan de forma no verbal y constituyen una forma más clara y confiable de comunicación que orienta integración con los demás (13), lo cual permite o valida las interpretaciones y preponderancia que se ha concedido en el estudio a la comunicación no verbal sobre el contenido de las respuestas verbales $(14,15,16)$.

Entre los hallazgos se destaca que el comportamiento emocional de los adolescentes institucionalizados no es acorde con lo esperado como normal para esta etapa, en la cual se ha considerado que son "raras entre los adolescentes conductas de apatía o indiferencia", pues este grupo canaliza sus emociones en forma de "rebeldía o agresividad pasiva", lo cual se reconoce como el "actinout" del adolescente, concepto que representa que los miedos, angustias, temores, ira y enojo no son vividas y expresadas de manera interna, sino que a cambio las proyectan hacia lo externo, es por ello que la depresión adolescente se manifiesta en una serie de conductas hostiles, agresivas $(17,18)$.

Podría decirse entonces que el comportamiento emocional de los adolescentes internados no coincide con lo esperado para la etapa pues la gama de emociones displacenteras o negativas acumuladas durante la internación de los adolescentes son sugestivas de la presencia de depresión tal vez reactiva dadas las condiciones en que se desarrollaron las primeras etapas de sus vidas (19).

Las emociones que presenta el adolescente internado se relacionan más con el comportamiento de "ostracismo" el cual obedece o es respuesta a la frustración en humanos, y las respuestas emocionales de las personas varían en una amplia gama de conductas que van desde comportamientos pro-sociales compensatorios hasta la agresión [...] cuando se presenta en conjunto con la pérdida de los refuerzos sociales tal y como ocurre en ocasión de la internación, lo cual se refleja en el estado emocional-efectivo de ambivalencia entre el deseo de pertenencia a un grupo (social, familiar o de otro orden) y el control de su individualidad $(20,21,22)$.

\section{CONCLUSIONES}

Se logró identificar que las emociones de los adolescentes respecto a su internación tiene una variación importante dependiendo del aspecto que se evalúe. Las principales emociones que tienen los adolescentes acerca de su internación son de tipo placentero debido a que la internación en el Centro de Orientación les concede seguridad y confianza en cuanto a su capacidad de supervivencia y bienestar físico expresado en satisfacción de sus necesidades básicas.

A nivel de sus emociones internas e íntimas 
sin embargo se identifica que predominan las emociones displacenteras entre ellas la indiferencia y apatía que no son consideradas como rasgo "normal" del adolescente.

En las relaciones con sus pares, se identificaron dos grupos de emociones dependientes al parecer de las experiencias afectivas positivas previas en su historia de vida; así mientras un grupo de los adolescentes entrevistados considera a sus pares como "su familia", otro grupo de entrevistados exhibe problemas de relaciones y desapego emocional con el resto de NNA en su misma situación.

\section{RECOMENDACIONES}

Examinar el manejo afectivo previo que se le ha brindado a los adolescentes que ingresan por primera vez al centro, de manera que se programen de forma personalizada las medidas que tienen como fin intervenir y favorecer su proceso de adaptación a la institución.

Reclasificar o retomar las razones que conllevan a que los adolescentes que viven en hogares sustitutos sean retirados de éstos y reingresados a internación; ya que gran parte de las razones que han motivado esta situación particular obedecen a los comportamientos considerados como normales en esta etapa de cambios.

Que así mismo como se aborda la preparación para las capacidades laborales y autosuficiencia previo a la edad de emancipación, se fomente a través de la interacción de los adolescentes internados y sus cuidadores, el uso de diferentes herramientas para lograr las habilidades sociales y así disminuir el impacto negativo de la internación sobre la esfera social de la salud; con ello de manera simultánea se lograría reducir las causales de frustración todo ello en pro de las demandas emocionales, de libertad controlada y autonomía que requiere la emancipación a la que se acercan estos adolescentes bajo la protección del Estado.

\section{REFERENCIAS BIBLIOGRÁFICAS}

1 Esnaloa I. El autoconcepto físico durante el ciclo vital. Anales de Psicología 2008;24(1):1-n/a.

2 Fuentes MdCP, Linares JJG, Jurado MdMM, Flores FJS, Martín ABB. Valores interpersonales relacionados con el perfil de agresor y víctima en adolescentes. International Journal of Psychology and Psychological Therapy 2015 06;15(2):229-239.

3 García MR. Interacción y emociones. La microsociología de Randall Collins y la dimensión emocional de la interacción social. Psicoperspectivas 2015;14(2):51-61.

$4 \quad$ Litichever C. Entre la calle, la escuela, y las instituciones: trayectorias institucionales de jóvenes. Revista Latinoamericana de Ciencias Sociales, Niñez y Juventud 2016 Jan;14(1):177-190.

5 Gómez R. Psiquiatría clínica: diagnóstico y tratamiento en niños, adolescentes y adultos. 3 ed. Bogotá D.C: Editorial Médica Panamericana, 2008. 853 p. ISBN: 978-958-8443-01-0. p. 270.

6 Juliana Sánchez Garzón, John JQ, Oscar VO. Retos de las familias en sociedades en transición. Revista CES Salud Pública 2011;2(1):45-55.

7 Fernández-Daza M,P., Fernández-Parra A. Problemas de comportamiento y competencias psicosociales en niños y adolescentes institucionalizados. Universitas Psychologica 2013 07;12(3).

8 Urbiola I, Estévez A. Dependencia emocional y esquemas desadaptativos tempranos en el noviazgo de adolescentes y jóvenes. Psicología Conductual 2015;23(3):571-587. 
9 Colorado YS. La Inteligencia Emocional como Factor Protector ante el Suicidio en Adolescentes. Revista de Psicología GEPU 2012 06;3(1):182-200.

10 Bianca dB, Wagner A. Adolescent transgressors and the weakening of their social network when they return to the community. Ciência y Saúde Coletiva 2009 Mar;14(2):557-566.

11 Rohner RP, Carrasco MÁ. Teoría de la aceptación-rechazo interpersonal (Ipartheory): bases conceptuales, método y evidencia empírica/interpersonal acceptance-rejection theory (Ipartheory): theoretical bases, method and empirical evidence]. Acción Psicológica 2014 $12 ; 11(2): 9-25$.

12 Berthelot $\mathrm{N}$, Ensink $\mathrm{K}$, Bernazzani O, Normandin L, Luyten $\mathrm{P}$, Fonagy

P. Intergenerational transmission of attachment in abused and neglected mothers: the role of trauma-specific reflective functioning. Infant Mental Health Journal 2015 Mar;36(2):200-212.

13 Rivera AMR, Velasco VEA. Evaluación del funcionamiento de una familia con un adolescente con Trastorno Afectivo Bipolar*. Pensamiento Psicologico 2009 Jan;5(12):161-174.

14 Castro PJ, Van dV, Burgos-Troncoso G, Meneses-Pizarro L, Pumarino-Cuevas N, Tello-Viorklumds C. Teorías subjetivas en libros latinoamericanos de crianza, acerca de la educación emocional. Revista Latinoamericana de Ciencias Sociales, Niñez y Juventud 2013 Jul;11(2):703-718.

15 Marín-Díaz V, Sánchez-Cuenca Formación en valores y cuentos tradicionales en la etapa de educación infantil. Revista Latinoamericana de Ciencias Sociales, Niñez y Juventud 2015 Jul;13(2):1093-1106.

16 Meilán JJ,G., Carro J, Guerrero C, Carpi A, Gómez $\mathrm{C}$. El efecto de memoria congruente con el estado afectivo: reconocimiento diferencial de palabras de tristeza y alegria. Anales de Psicología 2012;28(1):266-n/a.

17 Borges A, Matos MGd, Diniz JA. Adolescent process and positive health: affective and cognitive scope. Psicologia, Reflexão e Crítica 2011;24(2):281-291.

18 López-Romero L, Romero E, Villar P. Relaciones entre estilos educativos parentales y rasgos psicopáticos en la infancia. Psicología Conductual 2012;20(3):603-623.

19 López-Romero L, Romero E. Rasgos psicopaticos y practicas educativas en la predicción de los problemas de conducta infantiles. Anales de Psicología 2012;28(2):629-n/a.

20 Pérez-González JC. Revisión del sentido y de los contenidos de la orientación personal. Revista Española de Orientación y Psicopedagogia 2010;21(2):434-442.

21 Arantzazu Rodríguez Fernández. La estructura tridimensional del bienestar subjetivo. Anales de Psicología 2011;27(2):327-n/a.

22 Moreno-Zavaleta M, Granada-Echeverri P. Interacciones vinculares en el sistema de cuidado. Revista Latinoamericana de Ciencias Sociales, Niñez y Juventud 2014 Jan; 12(1):121-139. 